\title{
Biomolecules at mineral surfaces: Structure-bonding-stability relationships
}

\author{
CARMEN ENID MARTINEZ ${ }^{1 *}$
}

${ }^{1}$ School of Integrated Plant Science, Cornell University, Ithaca, NY 14853, USA ("correspondence: cem20@cornell.edu)

The retention of biomolecules at mineral surfaces impacts the environmental cycling of nutrients and contaminants, as well as the development of mineralstabilized organic matter. Here we present the results from a series of studies probing the kinetics, equilibrium and bonding mechanisms associated with the sorption-desorption behavior of biomolecules (protein, DNA, pectin, LMWO) at mineral surfaces. We will show how the activity (e.g., enzymatic activity) of biomolecules (large and small) might be affected by their interaction with mineral surfaces, and as a result of organic-organic interactions both in solution and at the mineral surface. We also asses the stability of biofilm formation across a variety of microbial phylogenies.

For example, spectra of experiments with solutions containing a herbicide and a peptide showed features indicating that both adsorbed onto goethite, with the peptide interacting with goethite via its $\mathrm{C}-\mathrm{H}$ backbone functional groups in addition to interaction via amide I and II functionalities (Fig. 1). Our data also suggest that, once at the surface, the herbicide and the peptide interact through $\mathrm{H}$ bonding.

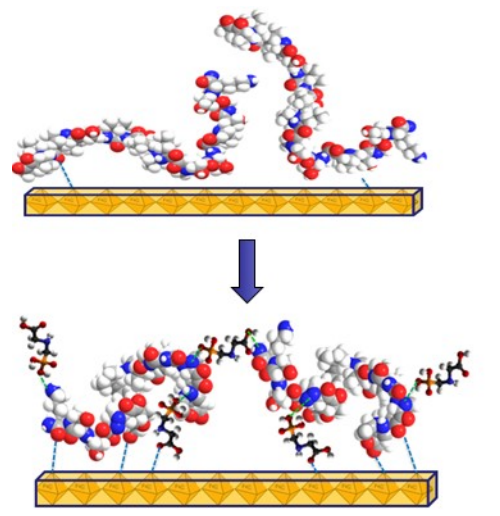

Figure 1.

Schematic: results

from an in situ

ATR-FTIR

(attenuated total

reflectance Fourier transform infrared) study of peptide and peptide-herbicide interactions at the goethite surface.

Links among structural characteristics, bonding environment, reversibilty and the stability of organic-mineral associations will be discussed. This work enhances our understanding of the molecular-level dynamics of biomolecule interactions at mineral surfaces. 